\title{
Severe malnutrition among children under the age of 5 years admitted to a rural district hospital in southern Mozambique
}

\author{
Tacilta Nhampossa ${ }^{1,2, *}$, Betuel Sigaúque ${ }^{1,2}$, Sónia Machevo 1,3 , Eusebio Macete ${ }^{1,4}$, \\ Pedro Alonso ${ }^{1,3}$, Quique Bassat ${ }^{1,5}$, Clara Menéndez ${ }^{1,5}$ and Victoria Fumadó ${ }^{5,6}$ \\ 'Centro de Investigação em Saúde da Manhiça (CISM), Vila da Manhiça, Rua 12, PO Box 1929, Maputo, \\ Mozambique: ${ }^{2}$ Instituto Nacional de Saúde, Ministerio de Saúde, Maputo, Mozambique: ${ }^{3}$ Faculdade de \\ Medicina, Universidade Eduardo Mondlane, Maputo, Mozambique: ${ }^{4}$ Direcção Nacional de Saúde, Ministerio \\ de Saúde, Maputo, Mozambique: ${ }^{5}$ Barcelona Center for International Health Research (CRESIB), Hospital Clínic, \\ Universitat of Barcelona, Barcelona, Spain: ${ }^{6}$ Hospital Universitari Sant Joan de Déu, Barcelona, Spain
}

Submitted 5 September 2012: Final revision received 6 March 2013: Accepted 15 March 2013: First published online 2 May 2013

\begin{abstract}
Objective: To describe the burden, clinical characteristics and prognostic factors of severe malnutrition in children under the age of 5 years.

Design: Retrospective study of hospital-based data systematically collected from January 2001 to December 2010.

Setting: Rural Mozambican district hospital.

Subjects: All children aged $<5$ years admitted with severe malnutrition.

Results: During the 10-year long study surveillance, 274813 children belonging to Manhiça's Demographic Surveillance System were seen at out-patient clinics, almost half of whom ( $47 \%)$ presented with some indication of malnutrition and $6 \%(17188 / 274813)$ with severe malnutrition. Of these, only $15 \%(2522 / 17188)$ were eventually admitted. Case fatality rate of severe malnutrition was $7 \%(162 / 2274)$. Bacteraemia, hypoglycaemia, oral candidiasis, prostration, oedema, pallor and acute diarrhoea were independently associated with an increased risk of in-hospital mortality, while malaria parasitaemia and breast-feeding were independently associated with a lower risk of a poor outcome. Overall minimum communitybased incidence rate was 15 cases per 1000 child-years at risk and children aged 12-23 months had the highest incidence.

Conclusions: Severe malnutrition among admitted children in this Mozambican setting was common but frequently went undetected, despite being associated with a high risk of death. Measures to improve its recognition by clinicians responsible for the first evaluation of patients at the out-patient level are urgently needed so as to improve their likelihood of survival. Together with this, the rapid management of complications such as hypoglycaemia and concomitant co-infections such as bacteraemia, acute diarrhoea, oral candidiasis and HIV/AIDS may contribute to reverse the intolerable toll that malnutrition poses in the health of children in rural African settings.
\end{abstract}

Keywords
Malnutrition
Bacteraemia
Risk factors
Children
Mozambique
In developing countries, malnutrition, with the different spectrum of diseases that it comprises, is highly prevalent and contributes significantly to the premature death of children. Malnutrition is believed to play a key role in up to a third of the 8.8 million annual deaths occurring in children under the age of 5 years, and malnourished children have a fourfold increased risk of death ${ }^{(1)}$. Significantly, sub-Saharan Africa carries the brunt of the impact caused by malnutrition, as almost half of its associated deaths occur there ${ }^{(2,3)}$.

Thus it appears that in order to decrease child mortality, one of the most relevant and pressing of the Millennium Development Goals, strategies to reduce both the prevalence and consequences of malnutrition will need to be put in place $^{(4)}$. Malnutrition is not a single disease, often underlies other conditions and comprises a wide spectrum of presentations that become evident principally as acute or chronic hindrances to the child's growth and development for a specific age. It arises from the combination of a series of intertwining environmental, nutritional, clinical, cultural and socio-economic determinants that need to be addressed in a comprehensive manner to reverse the vicious circle that leads to clinical disease. Clinical management of severely malnourished children is complex and challenging, requiring long hospitalization and a multidisciplinary approach. Updated WHO case management guidelines ${ }^{(5)}$ have been 
implemented in some African hospitals, but as a result of the existing weak and fragile health infrastructures, and the lack of specialized training to deal with such a complex condition, the management of malnutrition is often inadequate. Furthermore, the spread of the HIV pandemic in Africa and its impact on the nutritional status of sick children have triggered a secondary epidemic of severe malnutrition. The pathophysiology among severely sick malnourished children with AIDS and their clinical response to the accepted WHO therapeutic guidelines may be different from those of children with primary severe malnutrition secondary to food shortage rather than HIV infection.

As a strategy to improve case management of children hospitalized with severe malnutrition, the Manhiça Health Research Center (CISM), the Africa Viva Foundation and district health authorities started in 1999 a collaborative programme which includes continuous training of health workers to improve malnutrition diagnosis, management and post-discharge follow-up, assists with the provision of nutritional support through therapeutic foods and has enhanced the existing infrastructure by building a malnutrition-specific ward. In the current paper we present an analysis of the burden, clinical characteristics and risk factors for adverse prognosis associated with severe malnutrition among all patients $<5$ years of age admitted to Manhiça's District Hospital (MDH) from 2001 to 2010.

\section{Materials and methods}

\section{Study site and population}

The current retrospective study is based on information collected between January 2001 and December 2010 at $\mathrm{MDH}$, the referral health facility for Manhiça district, a rural area in southern Mozambique with about 140000 inhabitants. The area has been described in detail elsewhere $^{(6)}$. The Centro de Investigação em Saúde de Manhiça (CISM) has been running a Demographic Surveillance System (DSS) ${ }^{(7)}$ in the area and a morbidity surveillance system at the $\mathrm{MDH}^{(8)}$. Almost half (49\%) of the population lives beneath the poverty line of less than \$US 1 per day. Agricultural subsistence is the main activity in the district, together with work at the large sugar factories in neighbouring Maragra and Xinavane. Maize, sweet potatoes, peanuts and cowpea are the staple foods. Thirty-four per cent of the families are led by women (C Sacoor, personal communication, 2010). During the study period, other ongoing nutritional interventions at the community level organized by local agricultural government-based structures included the distribution and planting of sweet potato and the community supplementation of Plumpy'Nut and soyabean targeting HIV or tuberculosis patients, both adults and children. The area is endemic for malaria and the community prevalence of HIV is among the highest in the world, with prevalence rates reaching as high as $40 \%$ in the district ${ }^{(9)}$.
Severe malnutrition is the fourth commonest cause of hospital admissions.

\section{Data collection and clinical management}

MDH is a 110-bed hospital including a sixteen-bed specific malnutrition ward and admits about 4500 children per annum. Standardized forms are routinely completed for all out-patients and in-patients, and include demographic, clinical and laboratory data. Weight is measured for all out-patients but height is measured only for admitted patients. Mid upper-arm circumference is not routinely registered. Malaria is screened in all febrile patients. On admission, a single blood culture is performed for all children under the age of 2 years and for older children with a temperature $\geq 39^{\circ} \mathrm{C}$ or with severe malnutrition or other signs of severe disease according to clinical judgement ${ }^{(10)}$.

Following national and WHO recommendations, empirical antimicrobial therapy is started on admission for children with severe malnutrition ${ }^{(11,12)}$, using as first line a parenteral combination of ampicillin plus gentamicin. Penicillin, cloramphenicol or ceftriaxone may alternatively be administered according to availability or clinical severity. Antibiotic therapy is re-assessed based on clinical response and blood culture results. According to standard recommendations, malnourished children also receive mebendanzole and are supplemented with vitamin A, multivitamins and Fe (from week 2 onwards).

Nutritional supplementation with enriched milk formulas (F75 and F100), or with a hospital-made mixture of milk plus oil and sugar, is administered according to standard protocols. Weight is measured and re-assessed daily by a trained nurse using a hanging scale (infants) or an electronic floor scale (older ones) so as to assess the evolution and calculate nutritional requirements. Scales are calibrated routinely. Additional solid feeding is introduced as soon as possible according to age, appetite and resolution of oedemas. All complications and conditions associated with malnutrition, including hypothermia, hypoglycaemia, dehydration, sepsis, shock or heart failure, are assessed and managed according to standard WHO recommendations ${ }^{(5)}$. Immunization status is revised and updated at discharge for all children if necessary.

\section{Clinical definitions}

Weight-for-age (underweight), weight-for-height (wasting) and height-for-age (stunting) $Z$-scores were calculated for each child admitted during the study period using standard deviations and US growth charts ${ }^{(13-15)}$. We defined the types of malnutrition according to the different parameters calculated (weight-for-age, weight-for-height and height-for-age) and divided the study population into three malnutrition groups: severe $(Z$-score $\leq-3)$, mild to moderate $(Z$-score $>-3$ and $<-1)$ or non-existent $(Z$-score $>-1)$. Anaemia was classified as severe if packed cell volume was $<25 \%$ in neonates or $<15 \%$ for 
other paediatric age groups or non-severe if between 25 and $42 \%$ in neonates or between 15 and $32 \%$ for older infants. Increased respiratory rate was defined according to age standard definitions ${ }^{(5)}$. Hypoglycaemia was defined as severe if glycaemia on admission was $<2.2 \mathrm{mmol} / \mathrm{l}$; or moderate if between 2.3 and $3.0 \mathrm{mmol} / \mathrm{l}$. Deep coma required a Blantyre coma score $\leq 2$. Dehydration was defined according to standard WHO protocols ${ }^{(16)}$. Bacteraemia required the isolation of pathogenic non-contaminant bacteria in the blood.

\section{Statistical analysis}

Questionnaires were double entered in FoxPro-designed databases version $2 \cdot 6$ and statistical analyses performed with the STATA statistical software package version $9 \cdot 0$. Due to the characteristics of the sample (for $14-18 \%$ of the cases, we were unable to define height-for-age or weight-for-height status owing to missing height data), analytical inference was restricted to the weight-for-age indicator only. Recurrence of severe malnutrition was calculated considering a lag period of $30 \mathrm{~d}$ after each episode of severe malnutrition with a confirmed previous favourable discharge (thus excluding transferred or absconding children from the hospital). Minimum community-based incidence rates (MCBIR) were calculated by referring malnutrition cases to population denominators, establishing the time at risk (child-years at risk (CYAR)), inferred from the DSS information. Negative binomial regression models were estimated to compare incidence rates between age groups or calendar years. Models were estimated with a random intercept to take into account repeated measures, since children can belong to several age categories or to several calendar years during the follow-up. Overall $P$ values for age and calendar year were calculated using the likelihood-ratio test. Person-time was excluded after the first episode of severe malnutrition. Case fatality rates (CFR) were calculated by considering children with a known outcome at discharge (death or discharged) and represent in-hospital mortality. Proportions were compared using the $\chi^{2}$ test or Fisher's exact test and odds ratios and $95 \%$ confidence intervals were estimated using logistic regression. Wilcoxon ranksum tests were used for non-parametric comparisons. A multivariate logistic regression analysis was performed to assess independent risk factors for death among severely malnourished cases, using an automated backward stepwise estimation. Given that $3 \%(72 / 2522)$ of the severely malnourished children admitted $\mathrm{MDH}$ were transferred to Maputo's Central Hospital, 7\% (169/2522) absconded from hospital prior to a discharge decision by the caring physician and that the dependent variable was the final outcome (dead/alive), only children with a known outcome were included in the analysis. All variables that were associated with death at a significance level of $P<0 \cdot 10$ in the univariate analysis were included in the multivariate model. The significance level for removal from the model was set at $P=0.06$ and that for addition to the model at $P=0 \cdot 05$.

\section{Results}

During the 10-year long study period, 274813 children from the DSS area aged $<5$ years visited at the out-patient department of MDH. Six per cent (17844/274813) were subsequently admitted to the hospital, $52 \%$ of whom were male.

Six per cent $(17188 / 274813)$ of all out-patients could be classified according to their weight-for-age as severely malnourished, but only a small proportion (14.6\%; 2522/ 17188 ) ended up being admitted (Figure 1). Almost half of all visiting children (47\%; 128652/274813) suffered from some degree of malnutrition (mild, moderate or severe). Table 1 presents the proportion of in-patients according to age group (older or younger than 24 months of age) classified according to type and degree of malnutrition.

Recurring admissions in malnourished children were frequent. Indeed, from the 2522 severe malnutrition episodes, 1576 children were admitted once, 281 children were admitted twice, sixty-two children were admitted three times and further thirty-eight children were admitted more than three times. Figure 2 presents absolute numbers of malnutrition cases and deaths according to calendar year of admission.

\section{Clinical features of children admitted with severe malnutrition}

The prevalence of severe malnutrition was $11 \%$ (626/ 5672) among hospitalized infants aged $<12$ months, $23 \%(1169 / 5158)$ for children aged 12-23 months, $14 \%$ (449/3169) for children aged 24-35 months and 10\% (278/2844) for children 36-59 months of age $(P<0 \cdot 001)$. Table 2 summarizes the clinical and demographic characteristics of the 2522 severely malnourished children $<5$ years of age admitted during the study period, and compares them with all other non-severely malnourished admissions to hospital in this same age group. Children admitted with severe malnutrition were significantly younger than other admissions (mean age $20 v$. 21 months, respectively; $P=0 \cdot 007)$. Admissions with severe malnutrition seemed to occur more frequently during the rainy season, the busiest period for the hospital, similarly to what occurred with the rest of admissions $(P=0 \cdot 008)$. Children with severe malnutrition also appeared more significantly ill, and reported symptoms and witnessed signs were generally more frequent among severely malnourished children than in those better nourished. With the exception of malaria parasitaemia, highly prevalent among severely malnourished patients (52\%) but significantly more frequent among non-severely malnourished patients $(65 \%, P<0 \cdot 001)$, coexisting morbidities (pneumonia, acute diarrhoea, severe anaemia or 


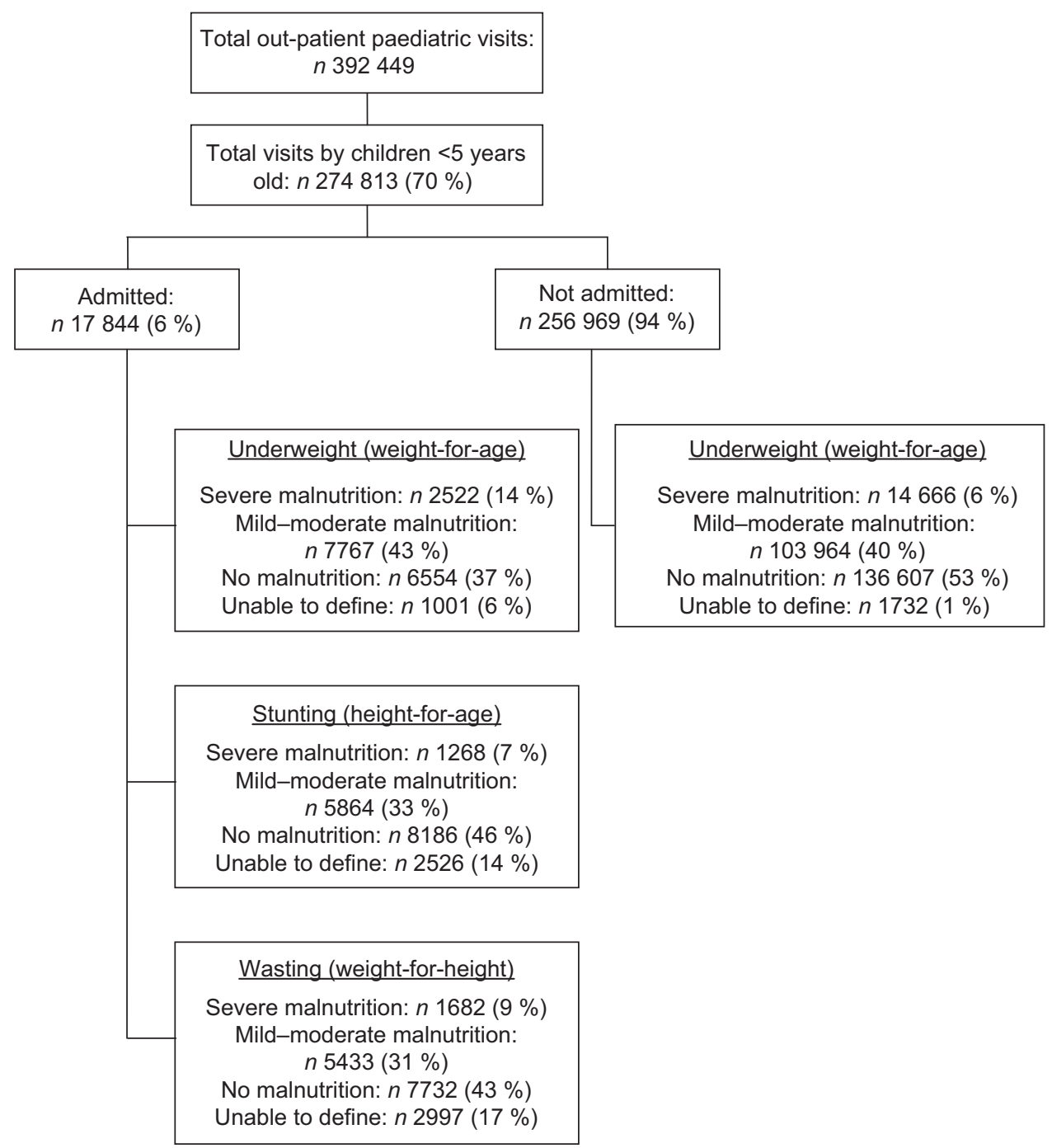

Fig. 1 Distribution of malnutrition among southern Mozambican children aged $<5$ years covered by Manhiça's Demographic Surveillance System, who visited as out-patients or were admitted to Manhiça District Hospital (from January 2001 to December 2010)

Table 1 Age distribution of malnutrition among southern Mozambican children aged $<5$ years covered by Manhiça's Demographic Surveillance System, who were admitted to Manhiça District Hospital (from January 2001 to December 2010)

\begin{tabular}{|c|c|c|c|c|c|}
\hline \multirow[b]{2}{*}{ Z-score } & \multicolumn{2}{|c|}{ Age $<24$ months } & \multicolumn{2}{|c|}{ Age $24-59$ months } & \multirow[b]{2}{*}{$P$} \\
\hline & $n / N$ & $\%$ & $n / N$ & $\%$ & \\
\hline \multicolumn{6}{|l|}{ Weight-for-age } \\
\hline$>-1$ & $4007 / 10830$ & 37 & $2547 / 6013$ & 42 & \\
\hline$<-1$ and $>-3$ & $5028 / 10830$ & 46 & $2739 / 6013$ & 46 & \\
\hline$\leq-3$ & $1795 / 10830$ & 17 & $727 / 6013$ & 12 & $<0.001$ \\
\hline \multicolumn{6}{|l|}{ Height-for-age } \\
\hline$>-1$ & $5824 / 10028$ & 58 & $2362 / 5290$ & 45 & \\
\hline$<-1$ and $>-3$ & $3534 / 10028$ & 35 & $2330 / 5290$ & 44 & \\
\hline$\leq-3$ & $670 / 10028$ & 7 & $598 / 5290$ & 11 & $<0.001$ \\
\hline \multicolumn{6}{|l|}{ Weight-for-height } \\
\hline$>-1$ & $4679 / 9561$ & 49 & $3053 / 5286$ & 58 & \\
\hline$<-1$ and $>-3$ & $3587 / 9561$ & 37 & $1846 / 5286$ & 35 & \\
\hline$\leq-3$ & $1295 / 9561$ & 14 & $387 / 5286$ & 7 & $<0.001$ \\
\hline
\end{tabular}

hypoglycaemia) were all significantly more frequent in the severe malnutrition group. Median duration of hospitalization in children was also significantly prolonged in severely malnourished patients (7 (IQR 3-9) $\mathrm{d} v$. 3 (IQR 2-5) d, $P<0 \cdot 001)$. CFR were significantly higher $(P<0 \cdot 001)$ for patients with severe malnutrition 


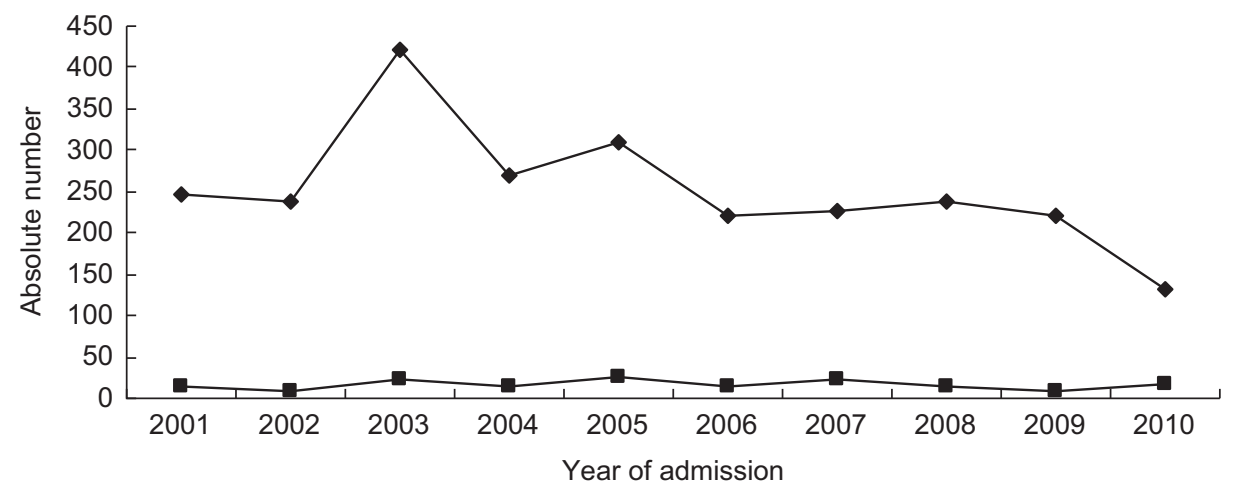

Fig. 2 Absolute numbers of annual admissions with severe malnutrition ( $-\longrightarrow)$ and related deaths (- - -) among southern Mozambican children aged $<5$ years, Manhiça District Hospital (from January 2001 to December 2010)

Table 2 Demographic and clinical characteristics of southern Mozambican children aged $<5$ years admitted to Manhiça District Hospital with severe malnutrition (from January 2001 to December 2010), compared with all other non-severely malnourished admissions in the same age group (mild and moderate malnutrition and non-malnourished)

\begin{tabular}{|c|c|c|c|c|c|}
\hline & \multicolumn{2}{|c|}{ Severe malnutrition ( $n$ 2522) } & \multicolumn{2}{|c|}{ Other admissions ( $n 14$ 321) } & \multirow[b]{2}{*}{$P$} \\
\hline & $n$ & $\%$ & $n$ & $\%$ & \\
\hline \multicolumn{6}{|l|}{ Demographic characteristics } \\
\hline \multicolumn{6}{|l|}{ Sex } \\
\hline Male & 1365 & 54 & 7920 & 55 & $0 \cdot 272$ \\
\hline Age (months) & & & & & \\
\hline Mean & \multicolumn{2}{|c|}{20} & \multicolumn{2}{|c|}{21} & \\
\hline SD & & & & & 0.007 \\
\hline \multicolumn{6}{|l|}{ Season } \\
\hline Rainy & 1573 & 62 & 8528 & 60 & 0.008 \\
\hline Breast-feeding ${ }^{\star}$ & 803 & 61 & 4825 & 79 & $<0.001$ \\
\hline \multicolumn{6}{|l|}{ Symptoms } \\
\hline Cough & 1836 & 73 & 9724 & 68 & $<0.001$ \\
\hline Reported breathing problems & 608 & 24 & 3104 & 22 & 0.006 \\
\hline Vomiting & 692 & 28 & 3355 & 23 & $<0.001$ \\
\hline Convulsions & 107 & 4 & 1263 & 9 & $<0.001$ \\
\hline Stop eating & 298 & 13 & 1212 & 9 & $<0.001$ \\
\hline Stop drinking or suckling & 199 & 8 & 842 & 6 & $<0.001$ \\
\hline \multicolumn{6}{|l|}{ Signs } \\
\hline \multicolumn{6}{|l|}{ Temperature $\left({ }^{\circ} \mathrm{C}\right)$} \\
\hline Mean & \multirow{2}{*}{\multicolumn{2}{|c|}{$\begin{array}{c}37 \cdot 7 \\
1 \cdot 3\end{array}$}} & \multicolumn{2}{|c|}{$38 \cdot 0$} & \\
\hline SD & & & & & $<0.001$ \\
\hline Pallor (mucosal) & 518 & 21 & 2350 & 16 & $<0.001$ \\
\hline Dehydration & 647 & 26 & 2174 & 15 & $<0.001$ \\
\hline Oedema & 298 & 12 & 524 & 4 & $<0.001$ \\
\hline Ear discharge & 110 & 4 & 374 & 3 & $<0.001$ \\
\hline Liver palpable & 101 & 4 & 392 & 3 & 0.001 \\
\hline Spleen palpable & 593 & 24 & 3768 & 26 & 0.003 \\
\hline Prostration & 281 & 16 & 1231 & 14 & 0.021 \\
\hline Deep coma (Blantyre coma score $\leqslant 2$ ) & 123 & 5 & 578 & 4 & 0.052 \\
\hline \multicolumn{6}{|l|}{ Co-morbidities } \\
\hline Malaria parasitaemia & 1258 & 52 & 9080 & 65 & $<0.001$ \\
\hline Pneumonia & 681 & 27 & 3282 & 23 & $<0.001$ \\
\hline Bacteraemia & 261 & 12 & 785 & 7 & $<0.001$ \\
\hline Acute diarrhoea & 784 & 31 & 2959 & 21 & $<0.001$ \\
\hline Severe anaemia & 312 & 12 & 1390 & 10 & $<0.001$ \\
\hline Hypoglycaemia & 133 & 6 & 534 & 4 & 0.034 \\
\hline Oral candidiasis & 155 & 6 & 248 & 2 & $<0.001$ \\
\hline \multicolumn{6}{|l|}{ Outcomes } \\
\hline Admission length $(\mathrm{d})$, mean and IQR & 7 & $3-9$ & 4 & $2-5$ & $<0.001$ \\
\hline Case fatality rate, $n / N$ and $\% \dagger$ & $162 / 2274$ & $7 \cdot 1$ & $222 / 13619$ & $1 \cdot 6$ & $<0.001$ \\
\hline
\end{tabular}

IQR, interquartile range.

${ }^{*}$ Denominators only include children aged $<24$ months.

tDenominators are different from total admitted patients in each group as they only include children with known outcome (absconded and transferred children excluded). 


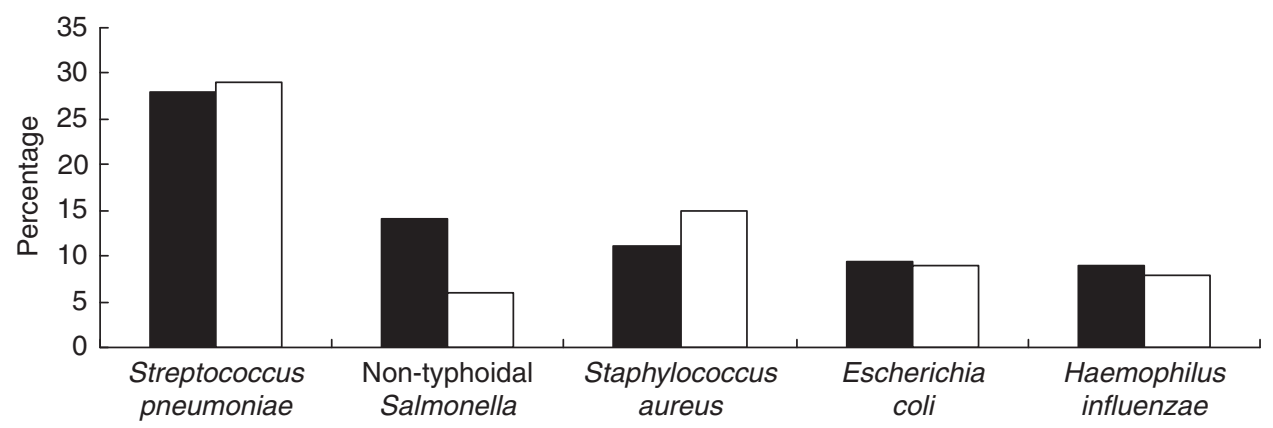

Fig. 3 Relative contributions of the main five bacterial isolates as causes of bacteraemia among severely malnourished ( $\mathbf{\square})$ southern Mozambican children aged $<5$ years, compared with all other non-severe malnutrition diagnoses ( $\square$ ), Manhiça District Hospital (from January 2001 to December 2010)

(162/2274; 7·1\%) when compared with all other nonseverely malnourished children (222/13619; 1.6\%) and importantly the risk of death seemed to increase in parallel with the degree of malnutrition (no malnutrition $v$. mild: $\mathrm{OR}=1 \cdot 16,95 \%$ CI $0 \cdot 83,1 \cdot 61$; mild $v$. moderate malnutrition: $\mathrm{OR}=1 \cdot 76,95 \mathrm{CI} \% 1 \cdot 26,2 \cdot 46$; moderate $v$. severe malnutrition: $\mathrm{OR}=2 \cdot 76,95 \%$ CI $2 \cdot 09, \quad 3 \cdot 63$; $P<0 \cdot 001)$.

The most common accompanying clinical diagnoses in children with severe malnutrition, as indicated by the discharging clinician, were malaria ( $52 \%$ ), acute diarrhoea (31\%), pneumonia (27\%), bacteraemia (12\%) and severe anaemia (12\%). Bacteraemia was significantly more common among children with severe malnutrition than among children without it ( $12 \% v, 7 \%$ in all other admissions; $P<0 \cdot 001$ ). Figure 3 shows the aetiology of bacteraemia in patients with severe malnutrition, as compared with the aetiology of bacteraemia for all other admissions. The most prevalent pathogens isolated from blood culture in malnourished children were Streptococcus pneumoniae (28\%), non-typhoidal Salmonella (14\%), Staphylococcus aureus (11\%), Escherichia coli (9\%) and Haemophilus influenzae (9\%). S. pneumoniae (28\% v. 29\%, P<0.001) and S. aureus $(11 \% v .15 \%, P<0 \cdot 001)$ were the only pathogens significantly less frequently isolated among severely malnourished children.

\section{Risk factors for poor outcome among admitted children with severe malnutrition}

Of the 162 deaths among severely malnourished children occurring during the study, $78 \%(126 / 162)$ occurred in children $<24$ months of age. CFR for severely malnourished children were significantly higher in the younger age groups: $12 \%(65 / 545)$ for infants, $6 \%(61 / 1068)$ for children aged 12-23 months and 5\% (36/661; $P<0 \cdot 001)$ for those aged $>24$ months. Independent risk factors for death among severely malnourished patients according to age group are shown in Table 3. Importantly, oral candidiasis and prostration independently increased the odds of death while malaria parasitaemia (both age groups) and breast-feeding (for those aged $<24$ months only)
Table 3 Independent risk factors for severe malnutrition death by multivariate analysis among southern Mozambican children aged $<5$ years, Manhiça District Hospital (from January 2001 to December 2010)

\begin{tabular}{|c|c|c|c|c|}
\hline \multirow[b]{3}{*}{ Variable } & \multicolumn{3}{|c|}{ Multivariate analysis } & \multirow[b]{3}{*}{$P$} \\
\hline & \multirow[b]{2}{*}{ OR } & \multicolumn{2}{|c|}{$95 \% \mathrm{Cl}$} & \\
\hline & & Lower & Upper & \\
\hline \multicolumn{5}{|c|}{ Children aged $<24$ months } \\
\hline Breast-feeding & 0.57 & 0.33 & 0.99 & 0.045 \\
\hline Prostration & $3 \cdot 20$ & $1 \cdot 77$ & $5 \cdot 80$ & $<0.001$ \\
\hline Oral candidiasis & 3.32 & $1 \cdot 70$ & $6 \cdot 48$ & $<0.001$ \\
\hline Hypoglycaemia & $2 \cdot 87$ & $1 \cdot 21$ & $6 \cdot 78$ & $0 \cdot 016$ \\
\hline Bacteraemia & 1.95 & 1.00 & $3 \cdot 78$ & 0.049 \\
\hline Malaria parasitaemia & 0.49 & $0 \cdot 26$ & 0.95 & 0.034 \\
\hline \multicolumn{5}{|c|}{ Children aged 24-59 months } \\
\hline Pallor & 3.35 & 1.05 & $10 \cdot 63$ & 0.041 \\
\hline Oral candidiasis & $20 \cdot 15$ & $2 \cdot 87$ & $141 \cdot 62$ & 0.003 \\
\hline Oedema & $10 \cdot 77$ & $2 \cdot 49$ & $46 \cdot 70$ & 0.001 \\
\hline Acute diarrhoea & $4 \cdot 39$ & 1.49 & $12 \cdot 91$ & 0.007 \\
\hline Prostration & $17 \cdot 38$ & $4 \cdot 36$ & $69 \cdot 20$ & $<0.001$ \\
\hline Malaria parasitaemia & $0 \cdot 30$ & 0.09 & 0.92 & 0.035 \\
\hline
\end{tabular}

were independently associated with a lower risk of a poor outcome.

\section{Minimum community-based incidence rates}

Table 4 show the age-specific MCBIR for severe malnutrition in the study area. Overall incidence was 15 cases per 1000 CYAR. The number of cases does not coincide with the numbers presented above, as MCBIR were calculated only for the first episode of severe malnutrition per child. During the study period, the incidence of severe malnutrition declined significantly from 33/1000 CYAR in 2001 to $7 / 1000$ CYAR in $2010(P<0 \cdot 001)$. The decline was higher in the period from 2001 to 2002 and mostly observed in the age groups 12-23 months and 24-59 months, in which it dropped by almost 50\%. The risk of severe malnutrition increased rapidly with age up to 24 months $(\mathrm{OR}=2 \cdot 23 ; 95 \% \mathrm{CI} 1 \cdot 94,2 \cdot 55)$, but then subsequently decreased ( OR $=0 \cdot 48 ; 95 \%$ CI $0 \cdot 40,0 \cdot 57$ ). MCBIR for severe malnutrition for the whole study period was 20/1000 CYAR in children aged 0-11 months 
Table 4 Minimum community-based incidence rate (MCBIR) of admitted severe malnutrition cases per 1000 child-years at risk (CYAR), by year of study, among southern Mozambican children aged <5 years, Manhiça District Hospital (from January 2001 to December 2010)

\begin{tabular}{|c|c|c|c|c|c|c|c|}
\hline Calendar year & Population & Cases & $\begin{array}{l}\text { Time at risk } \\
\text { (CYAR) }\end{array}$ & $\begin{array}{c}\text { MCBIR } \\
\text { (cases per } 1000 \text { CYAR) }\end{array}$ & IRR & $95 \% \mathrm{Cl}$ & $P$ \\
\hline 2001 & 8525 & 212 & $6453 \cdot 1$ & $32 \cdot 85$ & $1 \cdot 00$ & - & $<0.0001$ \\
\hline 2002 & 15139 & 171 & $9115 \cdot 6$ & $18 \cdot 76$ & 0.62 & $0.50,0.77$ & \\
\hline 2003 & 16520 & 298 & 12329 & $24 \cdot 17$ & 0.92 & $0 \cdot 75,1 \cdot 12$ & \\
\hline 2004 & 16474 & 199 & 12514 & $15 \cdot 90$ & 0.67 & $0.54,0.84$ & \\
\hline 2005 & 18269 & 222 & 13720 & $16 \cdot 18$ & 0.67 & $0.53,0.83$ & \\
\hline 2006 & 18631 & 182 & 14164 & $12 \cdot 85$ & 0.52 & $0.41,0.65$ & \\
\hline 2007 & 19005 & 190 & 14493 & $13 \cdot 11$ & 0.50 & $0.40,0.63$ & \\
\hline 2008 & 19364 & 189 & 14792 & $12 \cdot 78$ & 0.49 & $0.39,0.61$ & \\
\hline 2009 & 19412 & 183 & 14867 & $12 \cdot 31$ & $0 \cdot 46$ & $0.36,0.58$ & \\
\hline 2010 & 19710 & 112 & 15061 & $7 \cdot 44$ & $0 \cdot 27$ & $0.21,0.35$ & \\
\hline TOTAL & 51778 & 1958 & 127507 & $15 \cdot 36$ & & & \\
\hline
\end{tabular}

IRR, incidence rate ratio.

$P$ from negative binomial regression model with random effects using the likelihood-ratio test. NB: convergence was not achieved; estimates are based on iterated maximization.

and 35/1000 CYAR in children aged 12-23 months. Children from 24 to 59 months of age had the lowest MCBIR (7/1000 CYAR; $P<0 \cdot 001)$.

\section{Discussion}

The present study aimed to investigate the burden, clinical characteristics and risk factors associated with death among out-patients and hospitalized Mozambican children with severe malnutrition. Surprisingly, the majority $(>75 \%)$ of those children seen as out-patients and classified as severely malnourished according to their weight-for-age $Z$-score were not admitted to hospital. As current recommendations suggest that all severely malnourished children should be admitted, these findings indicate a massive failure to correctly identify paediatric malnutrition at the first encounter of the patients with the health facility ${ }^{(17,18)}$. In rural areas of sub-Saharan Africa, this first contact usually relies on health personnel with limited medical training and is a critical moment for the correct recognition of a common condition that entails an unacceptably high CFR $(>7 \%$ in our series). This very limited recognition and admission of severely malnourished children represents a missed opportunity to identify children who could benefit significantly from measures to increase their likelihood of survival, and calls for an immediate need for training of health personnel in the screening and identification of signs and symptoms associated with severe malnutrition. Even though many of these patients may have consulted with banal diseases, lack of recognition surely did prevent them from receiving appropriate management and nutritional advice to improve their derisory nutritional status.

The high prevalence (6-14\%), high associated CFR (7\%), clinical features and seasonal pattern of severe malnutrition found in the present study seem in general agreement with those described for other neighbouring areas $^{(10,19-22)}$. CFR among hospitalized children increase with increasing severity of malnutrition, peaking at about $7 \%$ for those children in the lowest malnutrition category ( $Z$-score $\leq-3$ ), and confirm the high morbidity and mortality burden associated with severe malnutrition in children under 5 years in the region and the massive underlying role that malnutrition plays among admitted patients, potentially impacting all-cause diagnoses on admission. Furthermore, it is also remarkable that 40-43\% of the children presented mild-to-moderate malnutrition. These results indicate a need for future efforts in identifying these children at risk of developing severe malnutrition at the first stages in order to provide adequate and timely preventive and therapeutic interventions.

Multivariate analysis showed that the presence of oedema, prostration and hypoglycaemia was each independently associated with an increased risk of death among severely malnourished children, suggesting that efforts should be made for an early screening of these complications at the health facility. While the diagnosis of oedema or prostration implies the recognition of simple clinical signs by health workers, identifying hypoglycaemia is clinically challenging as it depends on the availability of more costly devices. Pallor, a severe anaemia sign, was also identified as an independent risk factor for death in children with severe malnutrition. Screening and prevention of anaemia should therefore be performed among all malnourished children when attending the out-patient services in rural areas, especially in malaria-endemic countries where this infection may also contribute significantly to anaemia. Although conflicting evidence surrounds the routine administration of $\mathrm{Fe}$ supplements for the prevention of anaemia in malaria-endemic areas and current recommendations suggest withholding Fe for at least 1 week after malnutrition treatment has been initiated ${ }^{(23)}$, anaemia remains one of the major risk factors for a poor outcome among severely malnourished children and measures to prevent and treat it should be a priority.

The preventive impact of breast-feeding on malnutrition is significant ${ }^{(24,25)}$ and is well established, similarly to 
what we have shown in our series, particularly when breast-feeding is exclusive in the first 6 months of life and continued with safe, appropriate and adequate complementary feeding up to 2 years of age or even beyond $^{(26,27)}$. Taking into account that data regarding the kind of breast-feeding (exclusive or mixed) and associated individual hygienic, socio-economic and sanitation conditions (all factors with an important impact on the risk of malnutrition) were not adequately collected, future studies should explore the effect of the above-mentioned variables on the health of Manhiça district's children.

Data on HIV/AIDS co-infection, already highly prevalent in Manhiça district at the time, were unfortunately not routinely collected during the period covered by the present retrospective study, and HIV serostatus is unknown for these patients. However, it is likely that HIV infection suspected by typical signs such as the presence of oral candidiasis - would have emerged as an important factor contributing to the development of malnutrition and, once established, as a prominent independent risk factor for death ${ }^{(28,29)}$. Thus, measures to prevent mother-to-child transmission of HIV may very well be critical to decrease the impact that this infection imposes in the nutritional status of children and the prognosis of malnourished children. Moreover, the concomitant presence of bacteraemia and acute diarrhoea was confirmed in our study as other independent risk factors for death among malnourished patients. Malnutrition per se is a well-established risk factor for infections ${ }^{(10,30)}$. This frequent complication, independently associated with a poor prognosis, supports the obligate addition of wide-spectrum antibiotic coverage in any patient admitted with a diagnosis of severe malnutrition, so as to cover the most frequent bacteria found in this specific group of patients which, as other studies have worryingly shown, are becoming increasingly resistant to first-line therapies ${ }^{(31)}$.

Conversely, the presence of malaria parasitaemia was shown in our series to be independently associated with a decreased risk of dying. The supposed protection conferred by malaria parasites among malnourished children is a highly controversial issue, the pathophysiological bases of which are difficult to understand. While previous studies have shown either no significant association between the two diseases ${ }^{(32-34)}$ or even an increased risk of malaria morbidity among malnourished children ${ }^{(35,36)}$, a controversial study from Papua New Guinea suggested that malnutrition may protect children from malaria ${ }^{(37)}$. Methodological differences related to malnutrition definitions and in the age of children included were proposed to explain the discrepant results between the aforementioned studies. In the current study, those children presenting with malaria parasitaemia may have had a lower CFR because the direct cause of admission (i.e. the malaria infection) responds rapidly to treatment, possibly causing an early discharge motivated by resolution of the malaria episode but without taking into account the outcome of the associated and possibly unresolved malnutrition episode.

We also found that the risk of severe malnutrition increased rapidly until 24 months of age and then subsequently decreased. Risk of death, however, decreased uniformly with increasing age. The type of nutrient requirements and the physiological processes in less mature children $(<24$ months) compared with older children may explain this pattern. Moreover, the former group is also more heterogeneous in terms of underlying aetiologies and pathophysiology than older children.

Clinical management of severely malnourished children requires a multidisciplinary approach and long hospitalization that includes an initial period beginning on admission to hospital and lasting until the child's condition has stabilized, usually after a minimum of $7 \mathrm{~d}$. Follow-up and post-discharge monitoring are also part of the malnutrition management and are critical to prevent recurrences, common in this spectrum of diseases. However, and most importantly, adequate preventive measures need to be put in place to guarantee that the vicious circle leading to malnutrition does not occur in the first place. Judging by the high burden of malnutrition reported in the present study, it is clear that the few ongoing governmental-organized preventive activities at the community level are insufficient. Moreover, although CISM and the Manhiça district health authorities have an intra-hospital long-standing collaboration that started in 1999 with a clear aim to improve the detection and management of admitted malnourished patients, our findings reveal important functioning failures and important limitations of the programme. First, although the median duration of hospitalization in children with severe malnutrition was $7 \mathrm{~d}$, a sufficiently long period for an appropriate initial phase treatment, an important proportion of the severely malnourished cases (7\%, $169 / 2522$ ) absconded from hospital prior to a discharge decision by the caring physician, possibly contributing to the highly frequent re-admissions. Furthermore, with the exception of an initial and final decrease of its incidence (as measured by MCBIR, calculated only for the first episode) coinciding with the first and tenth years of the programme's activities, no subsequent significant variations in malnutrition incidence rates could be observed in the intervening years of programme activities. Additionally, absolute numbers of severe malnutrition cases and related deaths remained similar during the programme's implementation period. As previously mentioned, the rampant HIV/AIDS pandemic, highly prevalent at the community level and with a clearer higher impact after year 2005, may have significantly worsened the clinical evolution and prognosis of these patients ${ }^{(6,38)}$. Altogether, these findings support the theory that malnutrition is a complex medical emergency requiring a multidisciplinary approach. Improvements in its incidence rely not only on the availability of a well-functioning and accessible public 
health system, but also on parallel enhancements in the community's socio-economic status. More extensive measures to combat poverty and hunger and promote social inclusion, as well as a wider application of community education targeting the prevention of malnutrition, are aspects to be urgently considered by national public health nutrition policy makers.

The present study has other methodological limitations worth mentioning, including the fact that it retrospectively looks at a 10-year long series of patients, a fact limiting the interpretation of results and the direction of causality associations. Prospective studies taking into account the nutritional status of children in the hospital are therefore suggested. Finally, although height should be measured at admission, 14-18\% of the children had an incorrect registration of this indicator. The unavailability of height and other anthropometric data for all patients made an accurate assessment of malnutrition difficult. However, weight-for-age remains a well-established and accepted methodology for evaluating nutritional status because of the difficulties related to the monitoring of other anthropometric data in routine clinical practice. Nevertheless, the development of strategies to educate and qualify professionals working in hospitals in order to enhance the value of current techniques to assess nutritional status in patients attending hospitals, focusing on the paediatric population, should be reinforced.

\section{Conclusion}

Severe malnutrition among admitted children in this area of southern Mozambique is common but frequently undetected, despite its associated high risk of death. Measures to improve its recognition by clinicians responsible for the first evaluation of patients at the outpatient level are urgently needed, so as to improve their likelihood of survival. Together with this, the rapid management of complications such as hypoglycaemia and concomitant co-infections such as bacteraemia, acute diarrhoea and HIV/AIDS may contribute to reversing the intolerable toll that malnutrition poses in the health of children in rural African settings.

\section{Acknowledgements}

Sources of funding: This work was supported by a Gulbenkian Foundation grant for predoctoral research ('Bolsas de doutoramento na área das doenças tropicais negligenciadas para licenciados do PALOP') to the corresponding author (T.N.). Conflicts of interest: The authors declare that they have no conflicts of interest. Ethics: Ethical approval was not required. Authors' contributions: T.N. and B.S. contributed equally to the study. B.S., T.N., S.M., E.M. and Q.B. were clinicians taking care of malnourished children throughout the 10-year long study period. T.N., P.A., C.M., E.M. and V.F. were involved in the design and management of the malnutrition ward at MDH. B.S., T.N., C.M., V.F. and Q.B. performed and interpreted the analyses. T.N., B.S., V.F. and Q.B. wrote the first version of the manuscript. All authors agreed upon the submitted version of the paper and concurred with the subsequent revisions submitted by the corresponding author. Acknowledgements: The authors thank all study participants (children and caregivers) and all workers of the CISM for their help in obtaining the data. They also thank the Centre for International Health Research in Barcelona for encouraging the realization of this project and the Africa Viva Foundation for funding activities related to the MDH malnutrition programme. Finally, the authors thank the district health authorities for their collaboration in the ongoing research activities in Manhiça district.

\section{References}

1. Black RE, Cousens S, Johnson HL et al. (2010) Global, regional, and national causes of child mortality in 2008: a systematic analysis. Lancet 375, 1969-1987.

2. Rajaratnam JK, Marcus JR, Flaxman AD et al. (2010) Neonatal, postneonatal, childhood, and under-5 mortality for 187 countries, 1970-2010: a systematic analysis of progress towards Millennium Development Goal 4. Lancet 375, 1988-2008.

3. Morris SS, Cogill B \& Uauy R (2008) Effective international action against undernutrition: why has it proven so difficult and what can be done to accelerate progress? Lancet $\mathbf{3 7 1}$, 608-621.

4. UNICEF (2004). Millennium Development Goals: 1. Eradicate extreme poverty and hunger. http://www.unicef.org/ mdg/poverty.html (accessed October 2012).

5. World Health Organization (2005) Pocket Book of Hospital Care for Children: Guidelines for the Management of Common Illnesses with Limited Resources. Geneva: WHO.

6. Bassat Q, Machevo S, O'Callaghan-Gordo C et al. (2011) Distinguishing malaria from severe pneumonia among hospitalized children who fulfilled integrated management of childhood illness criteria for both diseases: a hospital-based study in Mozambique. Am J Trop Med Hyg 85, 626-634.

7. Nhacolo A, Nhalungo D, Sacoor C et al. (2006) Levels and trends of demographic indices in southern rural Mozambique: evidence from demographic surveillance in Manhica district. BMC Public Health 6, 291.

8. Guinovart C, Bassat Q, Sigauque B et al. (2008) Malaria in rural Mozambique. Part I: children attending the outpatient clinic. Malar J 7, 36

9. Gonzalez R, Munguambe K, Aponte J et al. (2012) High HIV prevalence in a southern semi-rural area of Mozambique: a community-based survey. HIV Med 13, 581-588.

10. Sigauque B, Roca A, Mandomando I et al. (2009) Communityacquired bacteremia among children admitted to a rural hospital in Mozambique. Pediatr Infect Dis J 28, 108-113.

11. Glisser M (editor) (1997) Normas Pediatricas. Maputo: Ministério da Saúde.

12. World Health Organization (2009) Handbook on Integrated Management of Childhood Illness (IMCI). Geneva: WHO.

13. Shann F (1993) Nutritional indices: Z, centile, or percent? Lancet 341, 526-527.

14. Kuczmarski RJ, Ogden CL, Grummer-Strawn LM et al. (2000) CDC growth charts: United States. Adv Data issue $314,1-27$. 
15. Cole TJ (1990) The LMS method for constructing normalized growth standards. Eur J Clin Nutr 44, 45-60.

16. Suh JS, Hahn WH \& Cho BS (2010) Recent advances of oral rehydration therapy (ORT). Electrolyte Blood Press $\mathbf{8}$, 82-86.

17. World Health Organization (1999) Management of Severe Malnutrition: A Manual for Physicians and Other Senior Health Worker. Geneva: WHO; available at http://www. who.int/nutrition/publications/malnutrition/en/

18. World Health Organization (2003) Guidelines for the Inpatient Treatment of Severely Malnourished Children. Geneva: WHO; available at http://www.who.int/nutrition/ publications/severemalnutrition/9241546093/en/index.html

19. Joosten KF \& Hulst JM (2008) Prevalence of malnutrition in pediatric hospital patients. Curr Opin Pediatr 20, 590-596.

20. Cartmell E, Natalal H, Francois I et al. (2005) Nutritional and clinical status of children admitted to the malnutrition ward, Maputo central hospital: a comparison of data from 2001 and 1983. J Trop Pediatr 51, 102-105.

21. Thakwalakwa C, Phuka J, Flax V et al. (2009) Prevention and treatment of childhood malnutrition in rural Malawi: Lungwena nutrition studies. Malawi Med J 21, 116-119.

22. Ashorn P, Phuka JC, Maleta K et al. (2009) Undernutrition malnutrition in infants in developing countries. Arch Pediatr Adolesc Med 163, 186.

23. Bhutta ZA, Ahmed T, Black RE et al. (2008) What works? Interventions for maternal and child undernutrition and survival. Lancet 371, 417-440.

24. Fawzi WW, Herrera MG, Spiegelman DL et al. (1997) A prospective study of malnutrition in relation to child mortality in the Sudan. Am J Clin Nutr 65, 1062-1069.

25. Briend A \& Bari A (1989) Breastfeeding improves survival, but not nutritional status, of 12-35 months old children in rural Bangladesh. Eur J Clin Nutr 43, 603-608.

26. Dadhich JP \& Agarwal RK (2009) Mainstreaming early and exclusive breastfeeding for improving child survival. Indian Pediatr 46, 11-17.

27. Alvarez-Uria G, Midde M, Pakam R et al. (2012) Effect of formula feeding and breastfeeding on child growth, infant mortality, and HIV transmission in children born to
HIV-infected pregnant women who received triple antiretroviral therapy in a resource-limited setting: data from an HIV cohort study in India. ISRN Pediatr 2012, 763591.

28. Saloojee H, De Maayer T, Garenne ML et al. (2007) What's new? Investigating risk factors for severe childhood malnutrition in a high HIV prevalence South African setting. Scand J Public Health Suppl 69, 96-106.

29. Kimani-Murage EW, Norris SA, Pettifor JM et al. (2011) Nutritional status and HIV in rural South African children. BMC Pediatr 11, 23.

30. Mandomando I, Macete E, Sigauque B et al. (2009) Invasive non-typhoidal Salmonella in Mozambican children. Trop Med Int Health 14, 1467-1474.

31. Mandomando I, Sigauque B, Morais L et al. (2010) Antimicrobial drug resistance trends of bacteremia isolates in a rural hospital in southern Mozambique. Am J Trop Med Hyg 83, 152-157.

32. Nyakeriga AM, Troye-Blomberg $\mathrm{M}$, Chemtai AK et al. (2004) Malaria and nutritional status in children living on the coast of Kenya. Am J Clin Nutr 80, 1604-1610.

33. Muller O, Garenne M, Kouyate B et al. (2003) The association between protein-energy malnutrition, malaria morbidity and all-cause mortality in West African children. Trop Med Int Health 8, 507-511.

34. Fillol F, Sarr JB, Boulanger D et al. (2009) Impact of child malnutrition on the specific anti-Plasmodium falciparum antibody response. Malar $J \mathbf{8}, 116$.

35. Friedman JF, Kwena AM, Mirel LB et al. (2005) Malaria and nutritional status among pre-school children: results from cross-sectional surveys in western Kenya. Am J Trop Med Hyg 73, 698-704.

36. Deen JL, Walraven GE \& von Seidlein L (2002) Increased risk for malaria in chronically malnourished children under 5 years of age in rural Gambia. J Trop Pediatr 48, 78-83.

37. Genton B, Al-Yaman F, Ginny M et al. (1998) Relation of anthropometry to malaria morbidity and immunity in Papua New Guinean children. Am J Clin Nutr 68, 734-741.

38. Naniche D, Bardaji A, Lahuerta M et al. (2009) Impact of maternal human immunodeficiency virus infection on birth outcomes and infant survival in rural Mozambique. $A m ~ J$ Trop Med Hyg 80, 870-876. 